\title{
Dynamic measurements of flowing cells labeled by gold nanoparticles using full-field photothermal interferometric imaging
}

Nir A. Turko

Darina Roitshtain

Omry Blum

Björn Kemper

Natan T. Shaked 


\title{
Dynamic measurements of flowing cells labeled by gold nanoparticles using full-field photothermal interferometric imaging
}

\author{
Nir A. Turko, ${ }^{a}$ Darina Roitshtain, ${ }^{a}$ Omry Blum, ${ }^{a}$ Björn Kemper, ${ }^{b}$ and Natan T. Shaked ${ }^{\mathrm{a}, \star}$ \\ ${ }^{a}$ Tel Aviv University, Department of Biomedical Engineering, Tel Aviv, Israel \\ bUniversity of Münster, Biomedical Technology Center, Münster, Germany
}

\begin{abstract}
We present highly dynamic photothermal interferometric phase microscopy for quantitative, selective contrast imaging of live cells during flow. Gold nanoparticles can be biofunctionalized to bind to specific cells, and stimulated for local temperature increase due to plasmon resonance, causing a rapid change of the optical phase. These phase changes can be recorded by interferometric phase microscopy and analyzed to form an image of the binding sites of the nanoparticles in the cells, gaining molecular specificity. Since the nanoparticle excitation frequency might overlap with the sample dynamics frequencies, photothermal phase imaging was performed on stationary or slowly dynamic samples. Furthermore, the computational analysis of the photothermal signals is time consuming. This makes photothermal imaging unsuitable for applications requiring dynamic imaging or real-time analysis, such as analyzing and sorting cells during fast flow. To overcome these drawbacks, we utilized an external interferometric module and developed new algorithms, based on discrete Fourier transform variants, enabling fast analysis of photothermal signals in highly dynamic live cells. Due to the self-interference module, the cells are imaged with and without excitation in video-rate, effectively increasing signal-to-noise ratio. Our approach holds potential for using photothermal cell imaging and depletion in flow cytometry. ๑ 2017 Society of Photo-Optical Instrumentation Engineers (SPIE) [DOI: 10.1117/1.JBO.22.6.066012]
\end{abstract}

Keywords: photothermal imaging; gold nanoparticles; interferometric phase microscopy; digital holographic microscopy.

Paper 170105RR received Feb. 17, 2017; accepted for publication May 25, 2017; published online Jun. 21, 2017.

\section{Introduction}

Photothermal (PT) imaging (PTI) of gold nanoparticles (AuNPs) has been developed as a molecular specificity approach for biomedical imaging in particular over the past decade. ${ }^{1-11}$ In PTI, AuNPs are first biofunctionalized to bind to specific targets, e.g., antigens on biological cells membranes and then excited optically for selective imaging. The PT effect is based on the transformation of light energy into heat. Under optical excitation with specific wavelength, AuNPs can form localized plasmonic resonance (LPR), an oscillation of the electron cloud around the particle at a resonance frequency. ${ }^{5}$ Depending on the size and shape of the nanoparticle, this frequency may be easily engineered to vary within the entire visible spectrum and into the mid-IR region, creating a narrow absorption spectrum. Thus, when illuminating the AuNPs with a wavelength that suits their plasmonic resonance, a strong absorption of light occurs and is followed by an energy release in the form of heat. This heat creates environmental effects, such as local refractive index changes that can be detected optically. It should be noted that under sufficiently high excitation intensities, AuNPs heating may cause structural damage to their surroundings.

In order to avoid temperature buildup, time-modulated pulsed excitation is used in PTI, where the excitation frequency can change from several $\mathrm{Hz}$ to $\mathrm{MHz}$. For this reason, PTI is not suitable for highly dynamic cells in flow, for which the dynamics

*Address all correspondence to: Natan T. Shaked, E-mail: nshaked@ post.tau. ac.il frequency range overlaps with the excitation temporal frequency. ${ }^{9}$ Thus, the motivation for increasing data acquisition and processing rate of PTI is clear. Fast PTI will allow a significant increase of throughput suitable for flow cytometry so PTI may become a common diagnostic tool, rather than limited to research.

Interferometric phase acquisition methods, such as differential interference contrast, ${ }^{1}$ optical coherence tomography (OCT), ${ }^{2,6,7}$ and digital holographic microscopy (DHM), ${ }^{9,11,12}$ are all capable of detecting optical phase changes induced by refractive index variations due to the temperature rise in the medium surrounding the heated AuNPs. OCT-based PTI has produced the best results so far, in terms of signal-to-noise ratio (SNR). However, OCT imaging is typically based on either scanning point-detection schemes or scanning line illumination, where both require costly equipment, especially when PTI of dynamic objects is needed.

In general, PTI techniques depending on scanning to obtain the full field of view (FOV) hold little potential in cost-effective imaging of dynamic objects. Moreover, in cases where the optically heated AuNPs change their position in the FOV during PTI, smeared images are formed, which affects visualization capabilities. In addition to the observation of cells during flow, the expansion of nonscanning PTI methods for usage with dynamic samples can be essential for capturing movements of tagged cellular organelles, tissue displacements, and thus, prospects to address applications which are currently hard to access by scanning-based PTI. We have recently proposed different methods for wide-field, scan-free PTI, based on our DHM

1083-3668/2017/\$25.00 @ 2017 SPIE 
systems. ${ }^{9,11}$ Since a large region of interest (ROI) is imaged by a single camera exposure, we are also able to image highly dynamic biological samples. ${ }^{13,14}$

Flow cytometry is a well-established method for highthroughput measurements and sorting of flowing biological cells, typically utilizing molecular specificity obtained by fluorescent biomarkers. To obtain high throughput, large amounts of cells flow through the device to produce large databases per sample in a short time. Imaging flow cytometry sacrifices throughput and cell flow velocity for better image quality and spatial data. ${ }^{15,16}$ For this goal, AuNPs hold a significant advantage over fluorescent biomarkers, which tend to photobleach, followed by signal degradation over time. ${ }^{17}$ AuNPs do not lose their efficiency before reaching significantly high excitation powers, and PTI keeps the same SNR during long courses of time. Moreover, AuNPs heating makes them attractive as specific bioagents for heating therapy.

Over the past decades, with the development of high-speed cameras, several methods for imaging dynamic cells have been developed. Some of them involve spectrally coded imaging ${ }^{18}$ and focus-stacking phase imaging. ${ }^{19}$ These methods use high frame-rate sensors of over 3000 frames per second and may capture from tens to thousands of cells per second, flowing at a speed of between 10 and $40 \mathrm{~mm} / \mathrm{s}$, significantly lower than flow cytometry but in the physiological range of blood flow. ${ }^{20}$

In this paper, we propose a simplified implementation of PTI for imaging cells in flow and new image processing principles for improved computational efficiency, with the potential for future integration in imaging flow cytometers. The new PTI processing approach increases the computation speed by decreasing the window size of the spectral analysis and a combination of the Goertzel discrete Fourier transform (DFT) with sliding DFT algorithms. ${ }^{21}$ Both approaches significantly increase the potential of PTI-based real-time imaging flow cytometry.

\section{Methods}

\subsection{Optical Setup}

For recording digital off-axis image holograms, we use a simplified interferometer ${ }^{22}$ that is illuminated by a low-temporalcoherence source. As shown in Fig. 1(a), our imaging beam is originated from a titanium:sapphire laser (Coherent, Micra$5, \lambda_{0}=780 \mathrm{~nm}, \Delta \lambda_{\mathrm{FWHM}}=10 \mathrm{~nm}$, up to $100 \mathrm{~mW} / \mathrm{cm}^{2}$, at the maximum imaging speed). The sample is illuminated by the condenser lens L1 $(f=300 \mathrm{~mm})$. The light that is transmitted by the sample is then magnified by a microscope objective $(40 \times, \mathrm{NA}=0.65$, Newport), passes the tube lens L2 ( $f=$ $150 \mathrm{~mm}$ ), and enters the self-interference module. ${ }^{16}$ This module interferes the image of the sample beam with its slightly shifted version to obtain an off-axis angle between the interfering beams, which is achieved by a slight tilt of one of the mirrors, so that the sample details in one beam overlap with empty locations in the shifted beam. The resulting interferograms are recorded by a CCD camera. Here, we choose to work with a resolution of $2 \mu \mathrm{m}$, which is enough to acquire PT signals from the cells, in order to image a large FOV. Note that our goal here is not to see the binding sites of the AuNPs on the cells but rather just to obtain PT from the labeled cells during fast flow.

The AuNPs excitation arm is comprised of a DPSS laser (Laserglow, $\lambda=532 \mathrm{~nm}$ ), which is expanded by a $1: 10$ beam expander (composed of lenses L3 and L4), and focused onto

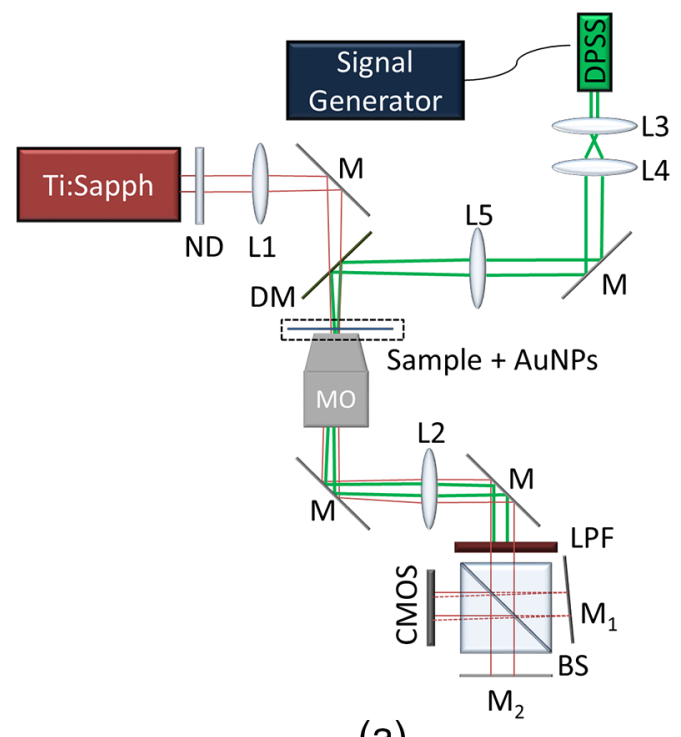

(a)

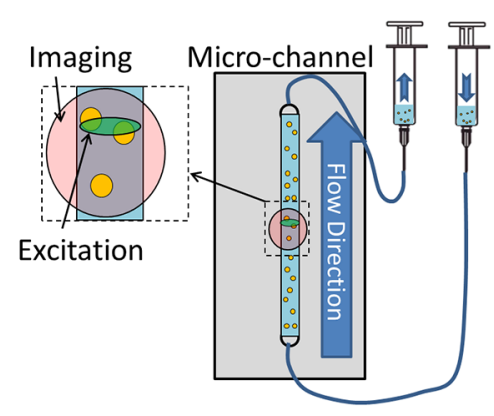

(b)

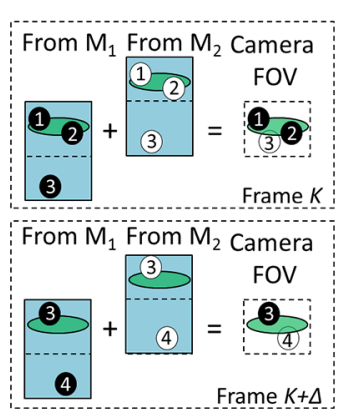

(c)
Fig. 1 Sample setup for dynamic PTI of cells in flow. (a) The PTI system, comprised of a simplified interferometric setup (red beams) and PT setup (green beam). In the interferometric path, a titanium: sapphire (Ti:sapph) laser beam is condensed by lens $L 1$ and imaged by $\mathrm{MO}$ and tube lens $\mathrm{L} 2$. Prior to reaching the sensor, the image enters an off-axis self-interference Michelson interferometer (BS, $\mathrm{M} 1$, and M2). In the PT excitation path, a signal generator modulates a DPSS laser (532 $\mathrm{nm}$ ), which is expanded and then focused on the sample plane. Later, a low pass filter LPF blocks the excitation beam from entering the interferometric module. (b) Top view of the flow chamber, placed in the sample plane. Two syringes with cells are coupled to the flow chamber and control the flow. The inset shows a zoom into the imaging and excitation beam location. (c) The arrangement of the interfering beams upon the camera plane, and circles 1 to 4 represent cells flowing in the channel.

the AuNPs-labled sample by L5 $(f 5=200 \mathrm{~mm})$, where AuNPs LPR excitation occurs. The excitation power on the sample is $33 \mathrm{~kW} / \mathrm{cm}^{2}$, which is independent of the imaging speed. The excitation beam is later filtered by long-pass filter LPF and is not detected by the camera. The light intensity of the DPSS laser source is temporally modulated by a signal generator, at frequencies lower than half the camera frame rate, to maintain proper sampling under Nyquist criteria for further spectral analysis.

\subsection{Sample Preparation and Cell Flow Experiment}

For PTI assessment of moving targets, we used the interferometric module to image cells flowing through a microfluidic channel. We used MDA-MB-468 breast cancer cells that express high levels of epidermal growth factor receptor (EGFR). AuNPs 
were synthesized and biofunctionalized by conjugating them with anti-EGFR antibodies (Sigma, Monocolonal Anti EGF Receptor, Clone 225). ${ }^{9}$

To label the cells with AuNPs prior to the experiment, cells were floated and incubated with a solution of the biofunctionalized AuNPs for $30 \mathrm{~min}$. After incubation, the solution was centrifuged at $1200 \mathrm{rpm}$ for $5 \mathrm{~min}$, and the pellet was extracted and placed in a syringe with $5 \mathrm{ml}$ phosphate buffered saline.

The flow setup comprised of input-output coupled syringes that controlled the suitable flow direction throughout the experiment. A flow chamber (Ibidi, $\mu$-slide, VI 0.1) and supplementary flow equipment were used to couple the syringes to the sample. Pressure was manually applied to the syringe in order to induce flow of the cells through the microfluidic channel. Both the imaging and excitation beams were aligned to fit the ROI within the microfluidic channel, positioned horizontally to keep gravity from disturbing the flow.

Since the self-interference method consists of the interference of two identical beams, both holding the same spatial information of the sample, the off-axis angle between them is chosen to be in the flow direction. Moreover, the angle was adjusted in such a way that it allows two different parts of the same ROI but in opposite phase, to appear simultaneously on the camera FOV, as they are reflected from the two mirrors as explained in Fig. 1 (c). With this alignment, we see the same cells moving twice as they flow through the FOV; in frame $k$, only cells 1 and 2 are excited by the excitation beam. Cell 3 appears on the sensor with an opposite phase, as its image is originated from M2. Therefore, although the phase profile of all cells will be visible (cells 1 and 2 will have positive phase and cell 3 will have negative phase), we will expect a PT signal around frame $k$ only from cells 1 and 2 . In frame $k+\Delta$, however, cell 3 reaches the excitation beam and is now imaged by M1. Around frame $k+\Delta$, cell 3 will produce PT signal, and cell 4, which is not excited, will not produce PT signal. This gives this configuration the advantage of viewing the same cell with and without excitation during the flow of the cells, for evaluation purposes, effectively creating an inherent control sample.

\subsection{Raw Data Acquisition}

The single-axis off-axis interferogram of the sample can be expressed as

$I=I_{M 1}+I_{M 2}+2 \sqrt{I_{M 1} I_{M 2}} \cos \left[\varphi(x, y)+\frac{2 \pi}{\lambda} x \sin \alpha\right]$,

where $I_{M 1}$ and $I_{M 2}$ are the intensities of the sample beams and its shifted version, respectively, $\varphi(x, y)$ is the phase difference between the beams, proportional to the sample thickness and the refractive index differences within the beam path, $\lambda$ is the wavelength, and $\alpha$ denotes the relative angle between both beams relative to the horizontal axis $x$. The second term of the cosine represents high-spatial-frequency phase modulation, dependent on $\alpha$, effectively acting as a spatial carrier frequency of the sample phase $\varphi(x, y)$.

The recorded off-axis interferogram is then digitally twodimensional Fourier-transformed and one of the cross-correlation terms is spatially filtered and centered. The argument of a reverse Fourier-transform of the result represents the phase of the sample, which can be unwrapped digitally to avoid $2 \pi$ ambiguities. ${ }^{23,24}$ The sample phase itself is a function of both the local refractive index differences $\Delta n$ and the physical thickness of the sample $\Delta z$. Therefore, changes in any of these parameters will be detectable by the DHM system. ${ }^{12}$

\subsection{Conventional PT Data Analysis and lts Problems in Imaging Dynamics}

For PTI, we used a time-modulated laser excitation that created plasmonic resonance around the AuNPs. The modulated excitation intensity induces time-modulated heat and a corresponding spectral phase signal while simultaneously avoiding heat accumulation. When analyzing the pixel-by-pixel spatial phase information, strong signals are present around the location of AuNPs. Two different approaches to view these phase changes may be used; the lock-in analysis, ${ }^{10}$ which is generally faster but less cost-effective and captures solely the PT signal, and spectral analysis of the full phase maps.

According to the spectral analysis approach, we perform a Fourier transform of the time-varying phase maps pixel-bypixel, binning the excitation frequency, and Fourier transform back, which results in a PT phase map indicating the location of the AuNPs in the sample. For nonstationary samples such as flowing cells, this analysis approach might fail. Flowing cells often show a broad frequency range that might overlap with the PTI excitation frequency and, thus, result in unwanted noise in the final PT phase map.

\subsubsection{Sliding DFT windowing for more efficient dynamic PTI}

The goal of dynamic PTI is to observe rapidly changing phase signals originating from nonstationary AuNPs that are dispersed within a sample. To optimize the processing, we only analyze a limited number of frames $N$ at every evaluation cycle, instead of processing all frames to form the PT image. This is enabled by the breakdown of an entire frame-by-frame temporally recorded sequence of PT images into smaller temporal subfractions (temporal windows); that allows the evaluation of data from a sample moving laterally during the phase sequence measurement.

From Parseval's theorem, we know that the spectrum noise floor of a signal is related to the number of sampled data points, in our case number of image frames, and as we reduce the number of processed frames per measurement, it results in poorer SNR values. Thus, there is a tradeoff between SNR and signal smearing, and there exists an optimal measurement time that minimizes movement smearing effects while keeping good SNR. This temporal window is specific for different optical setups and is influenced by the sensor recording speed, excitation frequency, sample velocity, and stationary signal SNR, but the more critical parameter is the lateral movement in pixels during a single excitation cycle. This pixel number per excitation cycle should be as low as possible, along with a sufficient sampling rate of the sensor. In this work, we have empirically tested the system performance for different window sizes. The PTI signal is derived from the excitation frequency value in the signal temporal spectrum, where only a single frequency bin needs to be further processed. Thus, there is no benefit in performing a full spectrum fast Fourier transformation (FFT). We therefore use the Goertzel algorithm ${ }^{15}$ in order to calculate the $k^{\prime}$ th bin for every window. The algorithm for finding the DFT value of variable $x$ at spectral bin $k$ is 


$$
\begin{aligned}
X(k)= & \sum_{n=0}^{N-1} x(n) e^{-j 2 \pi n k / N} \\
= & x(0)+x(1) e^{-j 2 \pi k / N}+\ldots \\
& +x(N-1) e^{-j 2 \pi(N-1) k / N}
\end{aligned}
$$

where $N$ is the window size, or the number of frames in our case, and $x(n)$ represents the phase values of frame $n$.

Lastly, recalculation at each time step of frame-sequence DFT is redundant. Adding a new frame to an existing windowed frame DFT does not force a whole new calculation. It is possible to reuse the data from the previous calculation to compute only the updated portion of information from the new frame. Thus, the spectral bin $k$, at the next frame will be

$$
\begin{aligned}
X_{n+1}(k)= & x(1)+x(2) e^{-j 2 \pi k / N}+\ldots+x(N-1) e^{-j 2 \pi(N-2) k / N} \\
& +x(N) e^{-j 2 \pi(N-1) k / N}
\end{aligned}
$$

where the index $n$ refers to the $n$ 'th frame. Equation (3) resembles the bin of the last frame, with a shift. We can look at

$$
\begin{aligned}
X_{n}(k) e^{j \pi k / N}= & x(0) e^{j 2 \pi k / N}+x(1)+x(2) e^{-j 2 \pi k / N}+\ldots \\
& +x(N-1) e^{-j 2 \pi(N-2) k / N}
\end{aligned}
$$

and can substitute Eq. (4) to Eq. (3) to obtain the final expression for the relation between both frames

$$
X_{n+1}(k)=\left[X_{n}(k)-x(0)\right] e^{j 2 \pi k / N}+x(N) e^{-j 2 \pi(N-1) k / N} .
$$

Note that in the resulting sliding DFT algorithm in Eq. (5), the number of calculations for each frame does not depend on the window size, which is highly beneficial for large frame sequences. This is accomplished by shifting the spectral values across the relevant frames of an evaluated series. Data of nonrelevant frames are dropped while values of new relevant frames are added. ${ }^{15}$ In short, when the value of the $k$ 'th bin is progressed by one frame, the new data replace the first frame of the previous window and all other data are multiplied appropriately to match Eq. (2).

The value of the parameter $k$ can be calculated according to

$k=\left|\frac{f_{\text {excitation }}}{\mathrm{FR}} N\right|$,

where $f_{\text {excitation }}$ is the excitation frequency and FR is the frame rate of the camera.

Another crucial factor for increased throughput capabilities is the maximum velocity of the cells during flow, which should be derived from the optical parameters of the system. If we require that an element of the size of a pixel in the sensor plane will not move more than one pixel during a single exposure, the following equation will describe the maximum blur-free cell velocity:

$$
v_{\max }=\frac{\mathrm{FR}}{N} \cdot \frac{p}{M}
$$

where $p$ is the camera pixel size and $M$ is the magnification of the imaging system. Note that the factor $1 / N$ is due to the temporal analysis that PTI requires. The factor accounts for the window size, during which any cell movement will cause blurring of the signal itself. These blurring artifacts can be removed as demonstrated in Sec. 3.3.

Equation (7) shows an inverse relation between the cell maximum velocity and the optical magnification. Thus, by using magnification of $5 \times$ (rather than $40 \times$ ), our system increases its throughput by a factor of 8 , similar to other existing imaging cytometers. It is noteworthy that the amount of data acquired by phase imaging is larger than bright-field microscopy, adding more information regarding the imaged cells; therefore, overall data throughput effectively increases as well.

\section{Results}

Due to our optical design, from each flowing cell we get two data sets. The first dataset is the PTI data, where the cell image is backreflected from mirror M1, corresponding to the time when it moves through the excitation beam and the AuNPs act as modulated heat sources, producing PT signals. Here, we expect a PT signal matching across the entire cell area. The second dataset, on the other hand, is where the cell image is formed from the backreflection from M2 and represents the time slot when it is not affected by the PT excitation. When looking at the phase images, both datasets can be easily distinguished, as the cells appear in opposite phase contrast.

When looking at the PTI images, we would expect the cells to show no PT signal when they are not excited. However, as mentioned in Sec. 2.3, the phase is proportional to the sample thickness and its refractive index, i.e., when a cell is moving across the ROI fast enough, with temporal frequency that overlaps with the PT excitation, it causes rapid changes in both parameters regardless of the PT excitation. This may create false-positive readings of cells, which are normally not expected to produce any PT signal, as well as cause smearing of the real PT signals of the marked cells. This is demonstrated in Fig. 2 (Video 1), where a nonspecific rise of the entire spectrum is evident when the nonexcited cell passes through the marked pixel. In contrast, the excited cell modulates the phase as expected to create a distinct PT signal in the excitation frequency. To reduce this unwanted effect, we divide the excitation frequency bin by another bin from the same frame, which greatly increased SNR.

\subsection{Algorithm Efficiency}

For dynamic measurements on moving objects with perspective real-time possibilities, fast processing capabilities are essential.

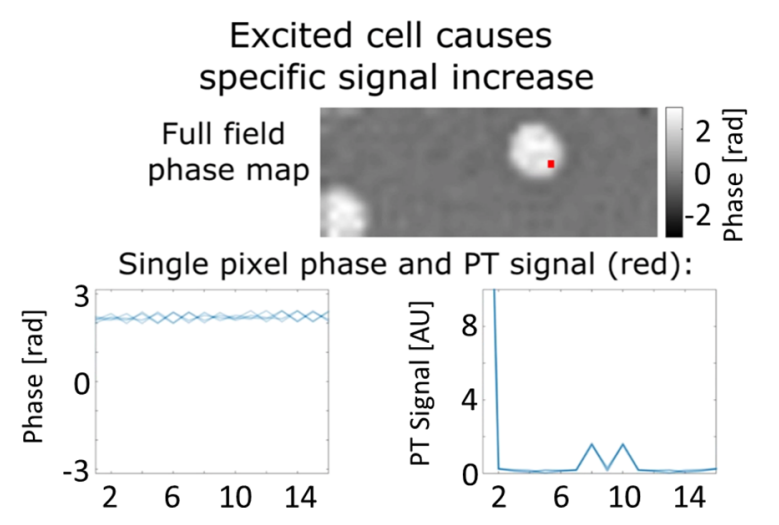

Fig. 2 Demonstration of PT signal specificity. (Video 1, MP4, 8.32 MB [URL: http://dx.doi.org/10.1117/1.JBO.22.6.066012.1]). 
(a)

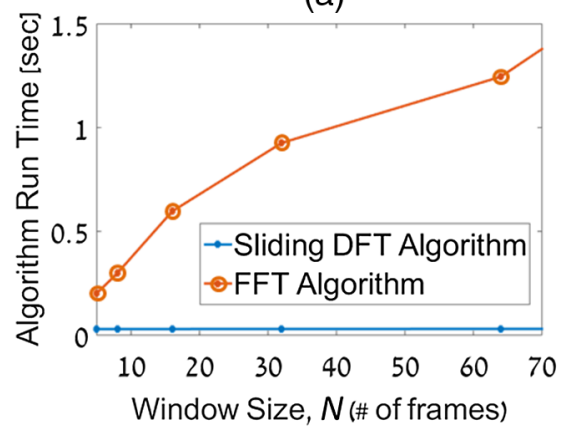

(b)

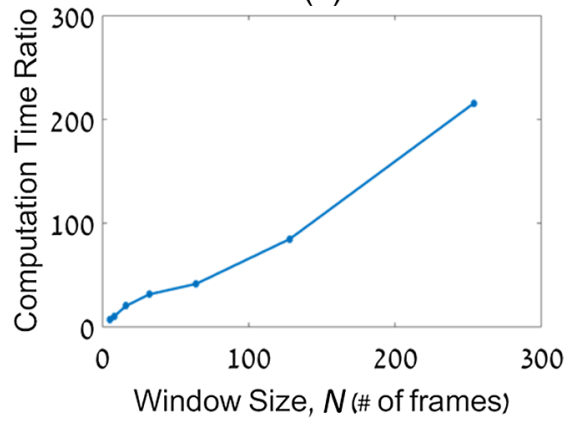

Fig. 3 Run time of (a) the sliding-DFT algorithm and conventional FFT algorithm and (b) the ratio between them (FFT/sliding-DFT) as a function of window size, averaged over 500 runs.

Therefore, we compared the runtimes of the suggested sliding DFT and the conventional FFT algorithms.

A set of 3500 phase maps of $13 \times 34$ pixels each, representing an ROI of $24 \mu \mathrm{m} \times 63 \mu \mathrm{m}$ within the FOV, was used for this comparison. These 3500 phase maps were analyzed by both algorithms for various window size $N$ with values ranging from 5 to 256 . Each configuration was run 500 times, and the average values of calculation times were compared, as shown in Fig. 3.

The runtime results for $N=5,8,16,32$, and 64 frames are shown in Fig. 3(a). While the algorithm based on performing an FFT on each window became slower when the number of frames increased, the sliding-DFT algorithm was evidently hardly affected by the number of frames. For $N=5$, the FFT average run time was $201 \mathrm{~ms}$, which was already too slow to produce video-rate calculation time (25 to $30 \mathrm{fps}$ ). In contrast, the sliding-DFT algorithm runtime for the same window size was $29 \mathrm{~ms}$, which is sufficient for video-rate processing of PT signals. For $N=64$ frames runtimes were 1240 and $30 \mathrm{~ms}$ for FFT and sliding-DFT algorithms, accordingly.

From Fig. 3(b), we can see an increase in the ratio of the FFT and sliding-DFT computation times as the number of frames increases. This trend demonstrates clearly the advantage of implementing sliding DFT in dynamic PTI, especially for a large number of frames. Using the sliding-DFT algorithm with a small window of five frames was 6.9 times faster than the conventional FFT algorithm, which represents a significant improvement. For a window size of 16 frames, already a ratio of 20 was measured, whereas for larger windows even several orders of magnitude of improvement were achieved.

\subsection{Windowing}

With large time windows, we get more data, hence enhancing the SNR, but the dynamic nature of the sample may cause smearing of the signal due to sample movement within that time frame. Therefore, we first qualitatively analyzed the signal appearance while changing the number of frames in the window in relation to frame rate and excitation frequency.

In order to test a wide range of window sizes $N$ without smearing the signal too much, we chose a relatively slow moving cell that moved at a speed of $200 \mu \mathrm{m} / \mathrm{s}$. The acquisition frame rate of the camera was $2000 \mathrm{fps}$ and the excitation frequency was set at $900 \mathrm{~Hz}$. In Fig. 4, we compare the PTI of the same frame as calculated with two different window sizes. As evident from Figs. 4(a) and 4(c), the signal is stronger and wider when we increase $N$, where a true signal should appear. From Figs. 4(b) and 4(d), however, we see that for a

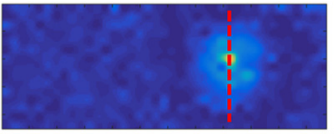

(a)

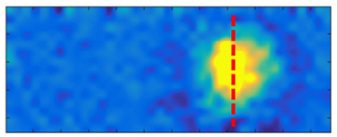

(c)

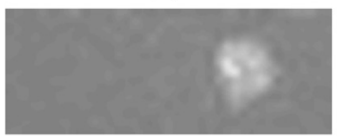

(e)

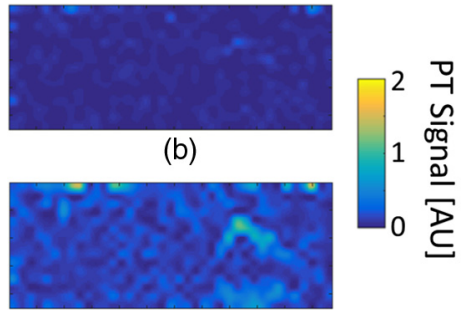

(d)

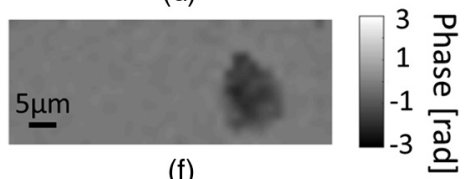

(f)
Fig. 4 PT signals at a temporal window size of $(a, b) N=8$ frames, (c, d), $N=64$ frames, and the corresponding quantitative phase images of the same cell as imaged by each mirrors individually. The excited cell is in (a, c, e) and the nonexcited cell with opposite (negative) phase values is in (b, d, f). Dashed red lines indicate the location of the cross-section graphs shown in Fig. 5.

larger window, a false signal appears at the edges of the moving cell, caused by the movement itself, as explained in Sec. 3.

The graphs in Fig. 5 show the cross-section of a PT true signal smear (in pixel units) and the signal rise as a function of window size. As expected, both the SNR [Fig. 5(a)] and smearing [Fig. 5(b)] increased for larger windows. Note that the large FWHM for $N=5$ that can be seen in Fig. 5(c) is caused by the low SNR and the proximity of the signal maximum to the noise floor. The exact optimal window size is specific for a certain system and an experiment setup. It is generally recommended to work with the smallest window size that still produces clear signals. For our measurements, we have found that $N=16$ achieves acceptable results.

\subsection{Dynamic PTI with Noise Reduction}

As proposed in Sec. 3, the false signals evident in Fig. 4(d) can be significantly reduced. This feature of the algorithm is shown in Fig. 6 (Video 2).

In this video, the brighter cells are the ones that are stimulated by the light of the excitation beam and are expected to form PTI signals, whereas the darker cells, or reference cells, are not influenced by the beam and should not form any distinct spectral signal on the excitation frequency. At first, only the excitation 
(a)

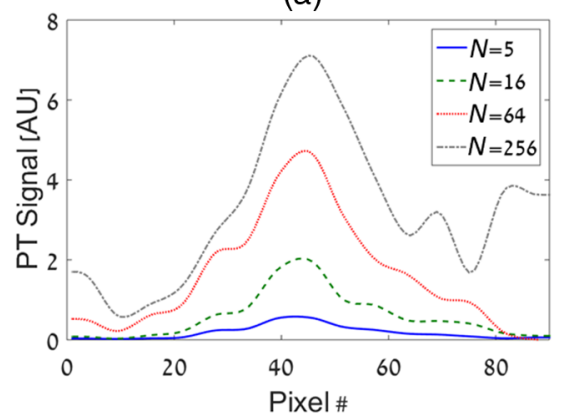

(b)

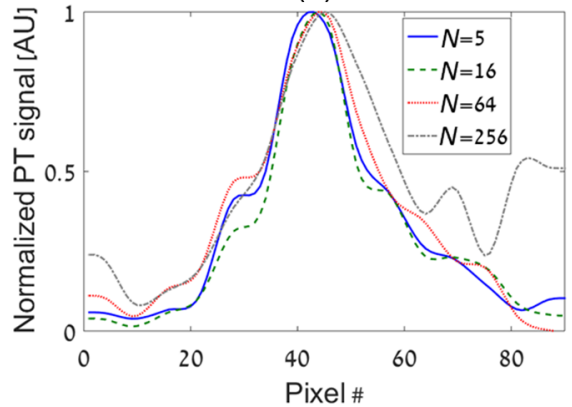

(c)

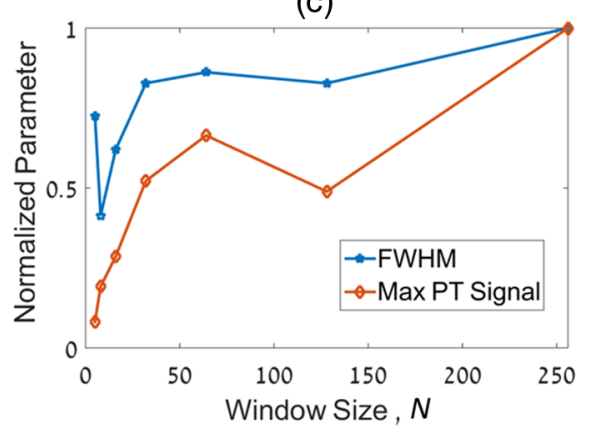

Fig. 5 Cross-sections of the PTI images at the middle of the cell, indicated by the dashed red line in Figs. 4(a) and 4(c), as calculated with different values of window size $N$. In (a), the PT signals shown are on the same scale, in (b) the self-normalized signals are shown. In (c), both the FWHM and maximum PT signal as a function of $N$, normalized to the maximal value, are shown.

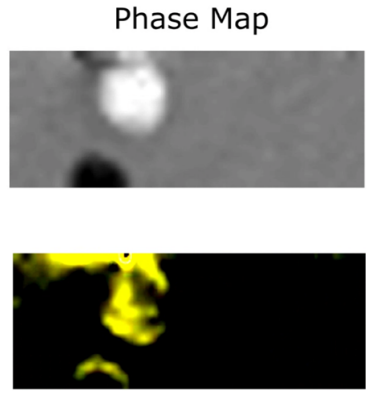

Dynamic PT signal

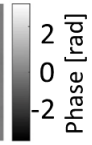

Noise-reduced PTI filters false signals

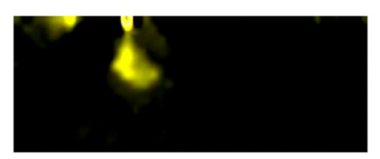

Noise-reduced dynamic PT signal

Fig. 6 Demonstration of noise reduction on dynamic PTI data. On the top image, white cells are cells under $\mathrm{PT}$ excitation and black cells are with no excitation. (Video 2, MP4, 10.9 MB [URL: http://dx.doi.org/10 .1117/1.JBO.22.6.066012.2]).

frequency bin is considered, and we do see strong "ghost" signals at the edges of the cells moving in the flow direction. These signals are more visible around the reference cells as they produce no real PT signal, but they do also appear on the excited cells as well. Since these signals are spectrally broadband, an increase of the excitation frequency spectral data is accompanied by a similar increase at the neighboring bins. Therefore, dividing the excitation frequency bin by a nonexcitation bin identified the real PTI signals that are originated by the presence of nanoparticles, rather than by the impact of cell flow. This is shown in the end of Video 2, where the reference cells pose no signal as a response to the excitation frequency.

\section{Summary and Conclusions}

Due to the growing interest in AuNPs and the prospects of using them as biomarkers, improved modalities are needed to explore

the LPR-based imaging capabilities that are formed by the particles. Full-field OCT and lock-in methods are capable of dynamic PT imaging of AuNPs but with a tradeoff of high cost. In this paper, we have presented a cost-effective system for full-field dynamic PTI of living cells in flow and an improved algorithm, which is based on a sliding window concept for fast data processing. This cost efficient interferometric phase microscopy approach allows the observation of living cells in flow and rapidly produces series of full phase maps of the entire FOV, in addition to the PT molecular-specific signals.

Our system is based on a simple microscope, with an interferometer add-on at the exit port and a PT excitation arm on the sample. The interferometer is used for phase acquisition of the dynamic samples while the excitation arm triggers the PT effect, which is manifested in phase changes retrieved from cells labeled with functionalized AuNPs. The principle of flowing cells through a microchannel in combination with an adapted alignment of the Michelson-interferometer module enabled the self-referencing concept, in which the same cells have been used as both target and control cells, depending on whether they have been under excitation or not in a certain FOV.

We have shown that for rapid dynamic imaging, it is necessary to analyze a smaller number of frames for each PT map as larger frame numbers may create artifacts of the signal such as smearing of a targeted cell, or a ghost signal of a nontargeted cell, resembling motion-blur in imaging. While the former artifact does not ordinarily affect the detection results of targeted cells, the latter might lead to false-positive readings. We succeeded in eliminating these ghost signals by spectral background deduction and thresholding. Other artifacts shown in Fig. 6 are background flashing, caused by medium flow, as well as actual waves of the liquid originated by the PT effect 
on the cells. This noise may be further reduced by implementing edge detection algorithms to separate cells from background.

Since every PTI system has its own optimal window size, depending on the target velocity, camera frame rate, and excitation frequency, we conclude that the well-known FFT algorithm, with computational complexity of the order of $N \cdot \log N$, can significantly limit the capabilities of the PT system for real-time imaging. Therefore, we implement a combination of algorithms that eliminates window size dependency frame-by-frame. The Goertzel algorithm is used to calculate only the necessary spectral data from the first $N$ frames. Then, for every frame we apply the sliding-DFT algorithm, the computational complexity is independent of $N$ and can only be related to the number of pixels. We have shown that using this algorithm, the computation time is almost independent of the window size and much faster than the FFT. We could demonstrate processing times in the range of $30 \mathrm{~ms}$ per frame while keeping a frame rate of over $30 \mathrm{fps}$.

The proposed system was set for feasibility studies regarding expanding PTI capabilities to dynamic imaging and has demonstrated $0.2 \mathrm{~mm} / \mathrm{s}$ flowing speed (using $2000 \mathrm{fps}$ ). However, recent imaging flow cytometry methods have the ability of acquiring data of thousands of cells per second with physiological velocities of 10 to $40 \mathrm{~mm} / \mathrm{s}$. As suggested by Eq. (7), the proposed system is potentially capable of acquiring PTI within these limits. Using an optical microscopy system with a magnification of $5 \times$, and camera pixel size of $20 \mu \mathrm{m}$, for example, while increasing the frame rate to $4000 \mathrm{fps}$, the imaging system will be able to handle cells flowing at $16 \mathrm{~mm} / \mathrm{s}$. It should be noted, however, that the trade-off between throughput and resolution should be taken into consideration per application. Further adjustments of the optical parameters of the system, using similar techniques utilized in imaging flow cytometry to increase throughput, ${ }^{25}$ are expected to allow faster flowing speeds in PTI as well.

This analysis is valid for fast acquisition of PTI for off-line efficient processing. Real-time PTI will still be limited to the processing power of the computer and algorithms, which may be further improved in the future. For instance, we may choose to analyze PT images of only a small ROI, or only choose relevant pixels after image segmentation to further improve the computational analysis time to be smaller than $30 \mathrm{~ms}$ per frame, or moreover using graphics processing units for parallel computing.

To conclude, with the emergence of AuNPs as contrast agents and PTI methods for specific molecular imaging, dynamic PTI capabilities are crucial for high-throughput measurements and flow cytometry-based classification of specific cell populations. Toward this goal, we have demonstrated that the proposed method is capable of dynamic, fast-processing PTI, and simultaneously provides rapid processing for regular quantitative imaging, which is essential for deriving cellular parameters, such as volume and dry mass. We thus expect that the proposed dynamic PTI concept will be integrated in future multimodal quantitative phase imaging systems, and will assist in providing specificity in phase imaging of rapidly changing samples, with an option for cell population depletion by PT treatment.

\section{Disclosures}

The authors have no relevant financial interests in this article. This study was funded by the German Israeli Foundation (GIF No. I-208-401.8-2013).

\section{References}

1. L. Cognet et al., "Single metallic nanoparticle imaging for protein detection in cells," Proc. Natl. Acad. Sci. U. S. A. 100(20), 11350 (2003).

2. D. C. Adler, R. Huber, and J. G. Fujimoto, "Phase-sensitive optical coherence tomography at up to 370,000 lines per second using buffered Fourier domain mode-locked lasers," Opt. Lett. 32(6), 626-628 (2007).

3. R. Shukla et al., "Biocompatibility of gold nanoparticles and their endocytotic fate inside the cellular compartment: a microscopic overview," Langmuir 21(23), 10644 (2005).

4. P. K. Jain et al., "Calculated absorption and scattering properties of gold nanoparticles of different size, shape, and composition: applications in biological imaging and biomedicine," J. Phys. Chem. B 110(14), 72387248 (2006).

5. V. M. Shalaev and S. Kawata, Nanophotonics with Surface Plasmons, pp. 219-228, Elsevier, Amsterdam and Oxford (2007).

6. M. C. Skala et al., "Photothermal optical coherence tomography of epidermal growth factor receptor in live cells using immunotargeted gold nanospheres," Nano Lett. 8(10), 3461-3467 (2008).

7. A. Gaiduk et al., "Detection limits in photothermal microscopy," Chem. Sci. 1(1), 343-350 (2010).

8. E. Absil et al., "Photothermal heterodyne holography of gold nanoparticles," Opt. Express 18(2), 780-786 (2010).

9. N. A. Turko, A. Peled, and N. T. Shaked, "Wide-field interferometric phase microscopy with molecular specificity using plasmonic nanoparticles," J. Biomed. Opt. 18(11), 111414 (2013).

10. W. J. Eldridge et al., "Fast wide-field photothermal and quantitative phase cell imaging with optical lock-in detection," Biomed. Opt. Express 5(8), 2517-2525 (2014)

11. N. A. Turko et al., "Detection and controlled depletion of cancer cells using photo thermal phase microscopy," J. Biophotonics 8(9), 755-763 (2015).

12. E. Cuche and C. Depeursinge, "Digital holography for quantitative phase-contrast imaging," Opt. Lett. 24(5), 291-293 (1999).

13. P. Girshovitz and N. T. Shaked, "Fast phase processing in off-axis holography using multiplexing with complex encoding and live-cell fluctuation map calculation in real-time," Opt. Express 23(7), 87738787 (2015).

14. O. Backoach et al., "Fast phase processing in off-axis holography by CUDA including parallel phase unwrapping," Opt. Express 24(4), 3177-3188 (2016)

15. D. Basiji and M. R. Ogorman, "Imaging flow cytometry," J. Immunol. Methods 423, 1-2 (2015).

16. D. A. Basiji et al., "Cellular image analysis and imaging by flow cytometry," Clin. Lab. Med. 27(3), 653-670 (2007).

17. M. A. Mycek and B. W. Pogue, Handbook of Biomedical Fluorescence, CRC Press, New York (2003).

18. T. Elhanan and D. Yelin, "Measuring blood velocity using correlative spectrally encoded flow cytometry," Opt. Lett. 39(15), 4424-4426 (2014).

19. S. S. Gorthi and E. Schonbrun, "Phase imaging flow cytometry using a focus-stack collecting microscope," Opt. Lett. 37(4), 707-709 (2012).

20. A. C. Burton, Physiology and Biophysics of the Circulation, 2nd Ed., Year Book Medical Publishers, Chicago (1972).

21. E. Jacobsen and R. Lyons, "The sliding DFT," IEEE Signal Process. Mag. 20(2), 74-80 (2003).

22. B. Kemper et al., "Simplified approach for quantitative digital holographic phase contrast imaging of living cells," J. Biomed. Opt. 16(2), 026014 (2011).

23. M. D. Pritt and D. C. Ghiglia, Two-Dimensional Phase Unwrapping: Theory, Algorithms, and Software, Wiley, New York (1998).

24. U. Schnars and W. Jueptner, Digital Holography: Digital Hologram Recording, Numerical Reconstruction, and Related Techniques, Springer, New York (2005).

25. D. B. Kay, J. L. Cambier, and L. L. Wheeless Jr., "Imaging in flow," J. Histochem. Cytochem. 27(1), 329-334 (1979).

Biographies for the authors are not available. 